Ann. Zootech., I975, 24 (2), 3I5-321.

\title{
INFLUENGE D'UN APPORT DE LYSINE DANS L'ALIMENT SOLIDE OU DANS L'EAU DE BOISSON SUR LES PERFORMANCES DE CROISSANCE DU LAPIN
}

\author{
M. COLIN, F. LEBAS et A. DELAVEAU \\ Station de Recherches sur l'Élevage des Porcs, \\ Centre national de Recherches zootechniques, I. N.R. A., \\ 78350 Jouy en Josas
}

\section{RÉSUMÉ}

Les auteurs ont étudié chez le Lapin la possibilité de supplémenter une ration déficiente en lysine par un apport de cet acide aminé dans l'eau de boisson.

Après 8 jours de préexpérience, 24 lapins sont répartis en 3 lots soumis à différents traitements. Le premier consiste en la distribution d'un aliment dont le taux de lysine est inférieur au besoin. Pour le second traitement, l'apport de lysine est sensiblement égal à ce besoin. Dans le troisième traitement, les animaux reçoivent le régime déficient en lysine, mais cet acide aminé est ajouté à l'eau de boisson à une concentration assurant une ingestion totale théorique de lysine identique à celle enregistrée dans le deuxième traitement.

L'apport de lysine supplémentaire améliore très fortement la croissance $(+50 \mathrm{p}$. 1oo) et l'efficacité alimentaire $(+20$ à $+30 \mathrm{p}$. roo) des animaux. Le troisième traitement permet des performances légèrement supérieures à celles enregistrées dans le cas de la distribution du régime équilibré en lysine, par suite d'une plus grande consommation d'eau donc de lysine. L'étude de l'évolution des consommations dans le temps montre que l'appétit du Lapin peut être très sensiblement modifié par la proportion de lysine dans le mélange alimentaire ingéré (liquides + solides).

\section{INTRODUCTION}

Dans des travaux précédents (Colin, 1973 et 1974) 1'un d'entre nous a estimé le besoin en lysine du lapin en croissance à o,55-0,65 p. Ioo du régime. Afin de tenir compte des variations de consommation des animaux, il est plus rigoureux d'exprimer le besoin en quantités ingérées quotidiennement : celui-ci serait alors compris entre 500 et $600 \mathrm{mg}$ de lysine par jour pour des 
animaux âgés de 5 à ro semaines. Toutefois, un tel mode de formulation du besoin est d'un usage pratique difficile, tout au moins tant que la lysine est apportée exclusivement par un aliment solide unique. Par contre, la distribution d'une solution aqueuse de lysine pourrait permettre à l'éleveur de mieux moduler l'apport total de lysine et de couvrir avec plus de précision le besoin des lapins.

En ce sens, il nous a semblé utile d'étudier la possibilité de supplémenter un régime déficient en lysine par apport de cet acide aminé dans l'eau de boisson.

\section{MATÉRIEI ET MÉTHODES}

\section{Animaux}

Vingt quatre lapins croisés (LEBAS, I975), des 2 sexes et, âgés de 5 semaines sont alimentés une semaine avec un régime préexpérimental; ils sont alors répartis entre les trois traitements expérimentaux. Les animaux sont logés dans des conditions déjà décrites, (Colın, ArkHURST et LEBAs, r973). Les abreuvoirs à surface d'eau libre sont reliés à des éprouvettes graduées permettant de connaître les consommations d'eau par lecture directe avec une précision de $\pm 5 \mathrm{~g}$.

\section{Régimes alimentaires}

Trois aliments semi-purifiés à base de tourteau de sésame ont été utilisés (tabl. I) en raison de la faible teneur en lysine des protéines de sésame. Les 2 régimes expérimentaux respectivement carencé ct équilibré en lysine sont identiques à ceux précédemment décrits par CoLIN en 1974.

\section{TABLEAU I}

Composition des régimes utilisés

\begin{tabular}{|c|c|c|c|}
\hline Régime & Carencé & Équilibré & $\begin{array}{l}\text { Préexpé- } \\
\text { rimental }\end{array}$ \\
\hline \multicolumn{4}{|l|}{ Composition centésimale } \\
\hline Tourteau de sésame $\ldots . \ldots \ldots \ldots \ldots$ & 38,2 & 38,2 & 38,2 \\
\hline Amidon de mais $\ldots \ldots \ldots \ldots \ldots \ldots$ & 32,88 & 33,33 & 33,11 \\
\hline Huile d'arachide $\ldots \ldots \ldots \ldots \ldots$. & 3 & 3 & 3 \\
\hline Paille de blé.............. & 20 & 20 & 20 \\
\hline C.M.V. (1) $\left.{ }^{1}\right) \ldots \ldots \ldots \ldots \ldots$ & 4 & 4 & 4 \\
\hline Glutamate de $\mathrm{Na} \ldots \ldots \ldots \ldots$. & 1,92 & 1,13 & 1,52 \\
\hline L-lysine $\mathrm{HCI}\left({ }^{2}\right) \ldots \ldots \ldots \ldots \ldots$ & 0,00 & 0,34 & 0,17 \\
\hline \multicolumn{4}{|l|}{ Composition chimique estimée } \\
\hline 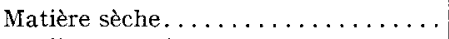 & 93,1 & 92,3 & 92,7 \\
\hline Matière organique............ & 82,3 & 81,8 & 82,0 \\
\hline Matières azotées $(\mathrm{N} \times 6,25) \ldots \ldots$. & 17,5 & 17,6 & 17,5 \\
\hline Cellulose $\ldots \ldots \ldots \ldots \ldots \ldots \ldots$ & 10,7 & 10,6 & 10,6 \\
\hline Teneur en lysine totale . . . . . . . . & 0,38 & 0,63 & 0,50 \\
\hline
\end{tabular}

(1) Colin, Arkhurst et Lebas, 1973.

(2) Produit commercialisé par Rousselot-Kuhlmann 95 p. 100 de L-lysine $\mathrm{HCl}$ à 80 p. 100 de lysine base (Brevet Ajinomoto, Tokyo). 
La lysine additionnelle de l'aliment équilibré a été fournie par du chlorhydrate de L-lysine en remplacement, sur une base isoazotée, d'une partie du glutamate de sodium du régime carencé.

La teneur en lysine de l'aliment préexpérimental est égale à la moyenne arithmétique dcs taux de cet acide aminé dans les deux régimes expérimentaux.

\section{Traitements expérimentaux}

Les deux premiers traitements consistent en la distribution à volonté de l'un des deux aliments expérimentaux. Dans le troisième traitement, les animaux reçoivent également ad libitum l'aliment carencé en lysine. Mais à partir du deuxième jour, de la lysine est ajoutée à l'eau de boisson à une concentration telle que ces lapins ingèrent au total approximativement la même quantité de lysine que ceux recevant l'aliment équilibré. La teneur en lysine de la solution est, pour chacune des 8 répétitions, fixée quotidiennement en supposant que pour le jour considéré la consommation d'aliment solide sera identique à celle de la veille et que les animaux boiront une quantité d'eau égale à la moyenne de celle des trois jours précédents. La solution nécessaire pour la conduite de l'expérience pendant 24 heures, est fabriquée chaque jour à partir de lysine en poudre.

Les lapins reçoivent l'aliment préexpérimental pendant 8 jours ; puis ils sont répartis entre les trois traitements pour une durée de ro jours.

\section{Contrôles effectués}

Les animaux sont pesés au début et à la fin de la période expérimentale. Leur consommation d'aliment est relevée quotidiennement par pesée des quantités distribuées et refusées. Les consommations d'eau sont lues - également quotidiennement — sur les éprouvettes graduées.

\section{Exploitation des résultats}

L'expérimentation est menée selon un schéma en blocs complets équilibrés. L'exploitation statistique des résultats est réalisée par une analyse de variance et par un test de Newman et Keuls (DaGNELIE, 1970 ).

\section{RÉSULTATS}

La supplémentation en lysine améliore très fortement les performances de croissance, de consommation et d'efficacité alimentaire (tabl. 2) des animaux. Les différences correspondant aux deux modalités d'apport de la lysine sont faibles et non significatives, sauf en ce qui concerne l'ingestion de liquide plus élevée pour les animaux ayant reçu de la lysine en solution $(237 \mathrm{~g}$ contre $207 \mathrm{~g} / \mathrm{j}$ ). La quantité totale de lysine ingérée est légèrement plus forte dans le troisième traitement que dans le deuxième. En fait, l'apport global de lysine correspond pour le troisième traitement à celui qu'assurerait un régime à $0,7 \circ \mathrm{p}$. Ioo de lysine.

L'étude de l'évolution des quantités d'aliment ingérées quotidiennement (fig. I) montre que le passage du régime préexpérimental à $0,5^{\circ} \mathrm{p}$. Ioo de lysine à celui apportant cet acide aminé au taux de 0,63 p. Ioo ne perturbe pas la consommation qui continue à croitre. Par contre, dans les deux autres traitements, les lapins qui ce jour précis ne reçoivent qu'un régime à o, 38 p. Ioo de lysine réduisent leur consommation par rapport à la veille de I 3 p. Ioo dans un cas, i 8 p. Ioo dans l'autre. L'addition de lysine à l'cau de boisson compense cet effet et la quantité d'aliment ingérée par les animaux devient le jour même pratiquement égale à celle observée dans le cas du régime équilibré. La consommation de liquide (fig. 2) crô̂t de $4 \mathbf{I}$ p. Ioo le jour où les animaux reçoivent la solution de Iysine et ensuite se stabilise approximativement au niveau ainsi atteint (230-270 g/jour). 
TABIEAU 2

Résultats zootechniqucs, après 10 jours d'expérimentation

\begin{tabular}{|c|c|c|c|c|}
\hline Critères & Régine carencé & $\begin{array}{l}\text { Régime } \\
\text { équilibré }\end{array}$ & $\begin{array}{c}\text { Régime carencé } \\
+ \text { lysine } \\
\text { dans l'eau }\end{array}$ & $\begin{array}{l}\text { Signification } \\
\text { statistique }\left(^{1}\right)\end{array}$ \\
\hline Lysine, p. 100 du régime ..... & 0,38 & 0,63 & $0,70\left({ }^{2}\right)$ & - \\
\hline Gain de poids $(g / j) \ldots \ldots \ldots$ & $32,2^{a} \pm 2,6\left({ }^{3}\right)$ & $47,2^{b} \pm 2,4$ & $\iota_{1} 9,5^{b} \pm 2,2$ & $* * *$ \\
\hline Indice de consommation $\ldots .$. & $3,35^{\alpha} \pm 0,16$ & $2,79^{b} \pm 0,13$ & $2,54^{b} \pm 0,08$ & $* * *$ \\
\hline Consommation d'aliment $(\mathrm{g} / \mathrm{j})$ & $106,1^{a}+5,3$ & $130,7^{b} \pm 5,7$ & $125,2^{b} \pm 5,1$ & $* *$ \\
\hline Consommation d'eau $(\mathrm{g} / \mathrm{j}) \ldots$ & $15^{4}, 0^{a}+12,1$ & $207, \mathbf{4}^{b} \pm 11,6$ & $237,5^{c} \pm 14,7$ & $* * *$ \\
\hline Lysine ingérée $(\mathrm{mg} / \mathrm{j}) \ldots \ldots \ldots$ & $400^{a} \pm 22$ & $819^{b} \pm 3 t$ & $917^{b} \pm 50$ & $* * *$ \\
\hline
\end{tabular}

$a, b, c$, : les valeurs d'une même ligne ayant en indice une lettre différente diffèrent significativement al seuil $P 0,05$.

(1) **: seuil 0,$01 ; \quad * * *$ : seuil 0,001 .

(2) $\frac{\text { Lysine ingérée }}{\text { Consommation d'aliment }}$.

(3) Ficart-type de la moyenne.

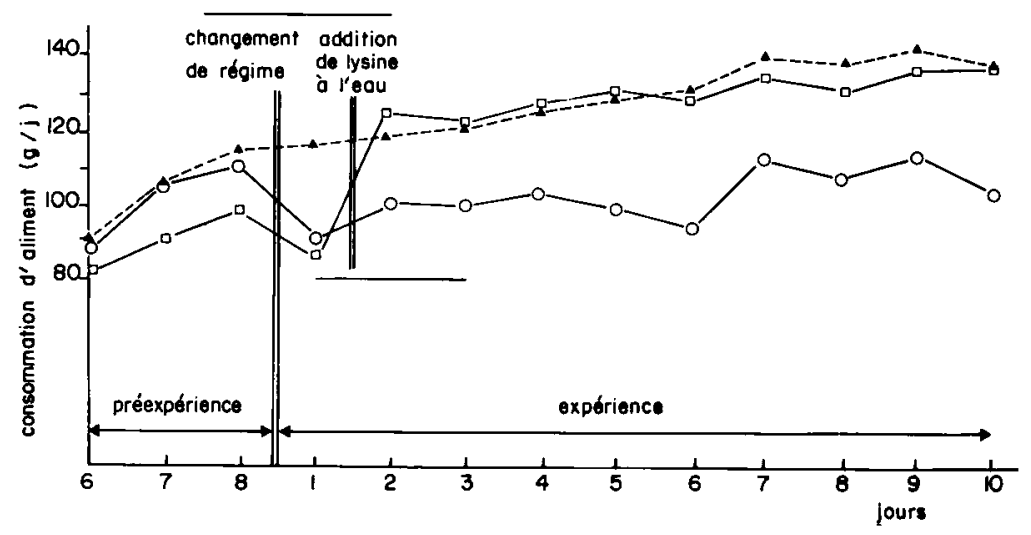

FIG. I. - Évolution dans le temps de la consommation d'aliment solide des lapins o-— régime carencé ;

A-..-- régime équilibré ;

$\square \longrightarrow \square$ régime carencé + lysine dans l'eau de boisson. 


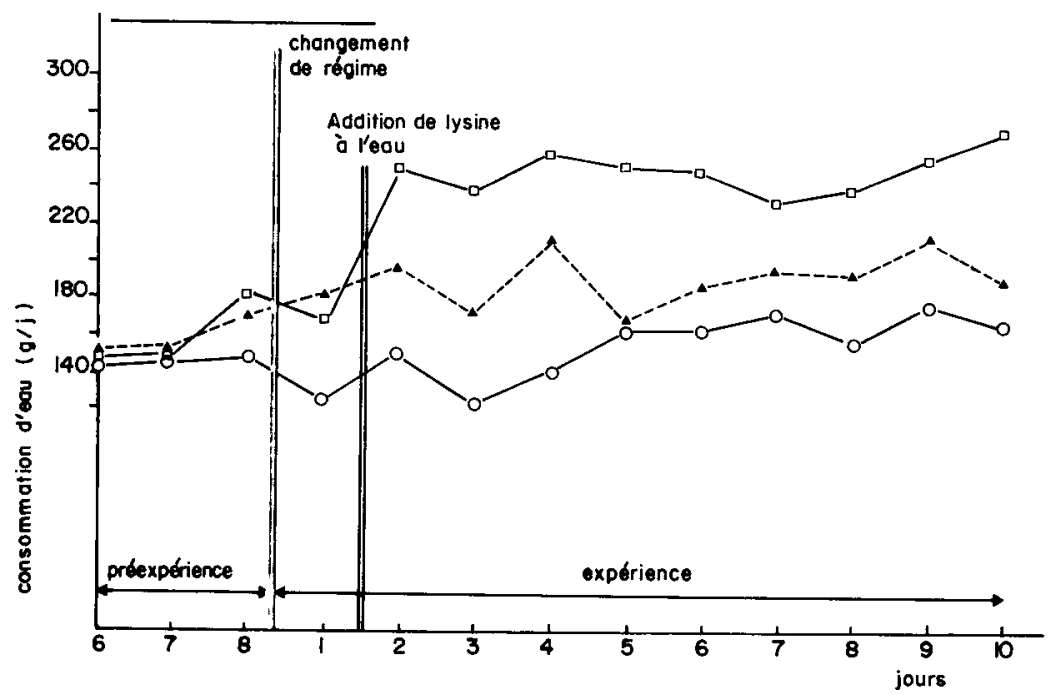

FIG. 2. - Evolution dans le temps de la consommation d'eau des lapins (voir légende de la fig. r)

\section{DISCUSSION}

Le niveau très élevé des performances enregistrées peut être partiellement attribué au fait que l'expérience a été menéc pendant les $7^{\circ}$ et $8^{\mathrm{e}}$ semaines de vie des animaux, qui correspondent pour le Lapin à une période de développement pondéral maximum (Prud'hon, Vezinet et Cantier, 1970).

Les résultats enregistrés confirment que l'addition de lysine à un régime déficient améliore très fortement la croissance et l'efficacité alimentaire des animaux (CoLrN, 1973 et 1974). Les deux formes d'apport de lysine permettent - à des doses approximativement identiques - des performances comparables. Les légères différences enregistrées en faveur des animaux recevant de la lysine en solution peuvent en partie s'expliquer par les difficultés rencontrées lors de l'extrapolation des consommations liquides et solides. Les lapins affectés à ce traitement ont effectivement ingéré roo $\mathrm{mg} /$ jour de lysine de plus que les autres. Or, si les recommandations pour l'apport journalier ont pu être fixées à $600 \mathrm{mg} /$ jour environ, il apparaît néanmoins que les gains et poids et les indices de consommation continuent à être légèrement améliorés pour des doses plus élevées (Colin, I974). Walstrom, Taylor et Seerley (I97o), Seerley et al., (r973) ont également signalé chez le Porc comme chez le Rat que l'apport de lysine dans l'aliment ou dans l'eau de boisson améliore de façon identique les performances de croissance. Ces derniers auteurs ont, de plus, montré qu'une solution de lysine conserve la même efficacité durant 3 I jours au moins. Cela nous permet d'admettre qu'une éventuelle dégradation de la lysine en solution ne peut être que négligeable pendant les 24 heures où nous l'avons utilisée.

L'évolution des consommations au cours du temps indique que l'appétit des lapins, pour un aliment solide équilibré par ailleurs, dépend de la proportion que représente la lysine dans le mélange alimentaire total ingéré (solide + liquide). Le sens de ces variations, la rapidité avec laquelle elles succèdent aux modifications de l'apport de lysine sont à rapprocher des phénomènes cités par HarPeR ( 1965 ) chez le Rat. D'après cet autcur, un déséquilibre par excès d'un ou plusieurs acides aminés essenticls déprime la consommation d'aliments en moins de 24 heures. Rose (r938) a également souligné que le retrait total d'un acide aminé essentiel entraîne rapide- 
ment une chute de l'appétit du Rat. Nos résultats semblent donc s'interpréter dans le cadre plus général de l'influence de l'équilibre en acides aminés essentiels de la ration sur l'appétit. Ces phénomènes peuvent s'expliquer soit par une action directe des acides aminés libres du sang sur le centre de la satiété (HARPER, I965), soit par une demande énergétique accrue, elle-même conséquence d'une augmentation de la protéinogénèse lors d'un apport supplémentaire de lysinc (RÉrat, 1971).

Par ailleurs, la brutale augmentation de la quantité de liquide que boivent les lapins, lors de l'addition de chlorhydrate de lysine à l'eau, montre que ces animaux sont très sensibles à la présence de ce corps. Cette observation diffère de celles réalisées chez le Porc ou le Rat qui modifient peu leur consommation d'eau (Walstrom, TAylor et SeErLEy et al., i 973). Il convient cependant de rester prudent dans l'interprétation de ce fait qui pourrait être provoqué par l'acide aminé lui-même aussi bien que par l'acide de chlorhydrique apporté par le monochlorhydrate de lysine.

En conclusion, une ration déficiente en lysine peut être supplémentée par un apport de cet acide aminé dans l'eau de boisson. Des lapins soumis à un tel traitement présentent des performances légèrement supérieures à celles obtenues avec un régime dont les protéines sont approximativement équilibrées en lysinc. L.e Lapin s'avère donc capable d'ajuster son ingestion d'éléments protéiques à partir d'une solution (pour la lysine tout au moins). Ce fait est à mettre en parallèle avec la capacité du Rat à maintenir sa consommation de protéines lorsque celles-ci sont apportées exclusivement par une solution d'hydrolysat de caséine à concentration variable (Rozin, I968).

Enfin, cette expérience a souligné les relations étroites existant entre le taux en lysine du mélange alimentaire ingéré et la consommation spontanée d'aliment solide par le Lapin.

Reçu par publication en décembre 1974.

\section{SUMMARY}

\section{INFLUENCE OF A SUPPLY OF LYSINE IN THE IOOD RATION OR IN THE DRINKING-WATER ON THE GROWTH PERFORMANCES OF RABBITS}

The possibility of supplementing a cleficient lysine diet with an addition of this amino acid to the drinking-water was studied in the rabbit.

After a pre-experimental period of 8 days, 24 rabbits were divided into 3 groups subjected to different treatments. In the first treatment, the lysine level of the diet was lower than the requirement. In the second one, the supply of lysine almost corresponded to the requirement and in the third one, the animals received the lysine deficient diet, but this amino acid was added to the drinking-water at a concentration theoretically ensuring a total lysine intake identical to that recorded in the second treatment.

The supplementary supply of lysinc highly improved growth ( $f-5^{\circ} \mathrm{p}$. IOo) and feed efficiency $(+20$ to $+30 \mathrm{p}$. Ioo $)$ of the animals. The third treatment led to slightly better performances than those recorded in the case of the lysine balanced cliet because of a higher water intake, and consequently a higher lysine intake. The stucly of the change of food consumptions in the course of time showed that the appetite of the rabbit may be noticeably modified by the proportion of lysine in the mixture of food ingested (liquid + solid).

\section{RÉFÉRENCES BIBLIOGRAPHIQUES}

Comin M., 1973. Influence de l'addition de lysine à des régimes à base de tourteau de sésame chez le Lapin. Journées Recherches Avicoles ct Cunicoles, I9-2 I, ITAV I éd., Paris.

Colis M., I974. Supplémentation d'un régime à base de tourteau de sésame chez le Lapin : effets sur les performances de croissance et le bilan azoté estimé par deux méthodes. Ann. Zootech., 23, Ir9-132. 
Colin M., Arkinurst G., Lebas $l^{i}$., I973. Effets de l'addition de méthionine au régime alimentaire sur les performances de croissance chez le Lapin. Ann. Zootech., 22, 485-49r.

Dagnelie P., I970. Théorie et méthodes statistiques, vol. 2, Ed. Duculot, S.A., Gembloux.

Harper A. E. in Muniso H. N., Allison J. B., r964. Mammalian protein metabolism, vol. 2, 87-134, Acad. Press New York, London.

LEBAS F., I975. Influence de la teneur en énergie de l'aliment sur les performances de croissance chez le Lapin. Ann. Zootech., 24, $28 \mathrm{I}-288$.

Prud'hon M., Vezinet A., Cantier J., i970. Croissance, qualité bouchère et coût de production des lapins de chair. B.T.I., 248, 203-2I2.

Rérat A., I97o. La valeur biologique des protéines: quelques acquisitions récentes. Ann. Zootech., 20, $193-246$.

Rose W. C., I938. The nutritive significance of the amino acids. Biol. Rev., 18, ro9-1 36.

Rozin P., I968. Are carbohydrate and protein intakes separately regulated? J.comp. physiol. psychol., 65, $23-29$.

Seerley R. W., Meeks C. E., Campbell H. C., Scarth R. D., i973. Effet of feeding lysine in solution to growing pigs and rats. J. Anim. Sci., 37, 9I-94.

Walstrom R. C., TAylor A. R., SEerley R., I970. Effects of lysine in the drinking water of growing swine. J. Anim. Sci., 30, 368-373. 